\title{
Oligonucleotide Delivery with Cell Surface Binding and Cell Penetrating Peptide Amphiphile Nanospheres
}

\author{
Didem Mumcuoglu, ${ }^{\dagger}$ Melis Sardan, ${ }^{\dagger}$ Turgay Tekinay, ${ }^{\ddagger}$ Mustafa O. Guler, ${ }^{* \dagger}$ and Ayse B. Tekinay ${ }^{*} \dagger$ \\ 'Institute of Materials Science and Nanotechnology, National Nanotechnology Research Center (UNAM), Bilkent University, 06800 \\ Ankara, Turkey \\ Department of Medical Biology and Genetics, Faculty of Medicine, Life Sciences Application and Research Center, Gazi University, \\ 06500 Ankara, Turkey
}

Supporting Information

\begin{abstract}
A drug delivery system designed specifically for oligonucleotide therapeutics can ameliorate the problems associated with the in vivo delivery of these molecules. The internalization of free oligonucleotides is challenging, and cytotoxicity is the main obstacle for current transfection vehicles. To develop nontoxic delivery vehicles for efficient transfection of oligonucleotides, we designed a self-assembling peptide amphiphile (PA) nanosphere delivery system deco-

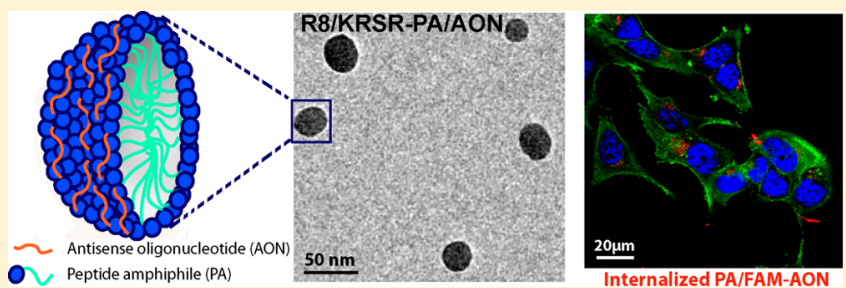
rated with cell penetrating peptides (CPPs) containing multiple arginine residues $\left(\mathrm{R}_{4}\right.$ and $\left.\mathrm{R}_{8}\right)$, and a cell surface binding peptide (KRSR), and report the efficiency of this system in delivering G-3129, a Bcl-2 antisense oligonucleotide (AON). PA/AON (peptide amphiphile/antisense oligonucleotide) complexes were characterized with regards to their size and secondary structure, and their cellular internalization efficiencies were evaluated. The effect of the number of arginine residues on the cellular internalization was investigated by both flow cytometry and confocal imaging, and the results revealed that uptake efficiency improved as the number of arginines in the sequence increased. The combined effect of cell penetration and surface binding property on the cellular internalization and its uptake mechanism was also evaluated by mixing $\mathrm{R}_{8}-\mathrm{PA}$ and KRSR-PA. $\mathrm{R}_{8}$ and $\mathrm{R}_{8}$ / KRSR decorated PAs were found to drastically increase the internalization of AONs compared to nonbioactive PA control. Overall, the KRSR-decorated self-assembled PA nanospheres were demonstrated to be noncytotoxic delivery vectors with high transfection rates and may serve as a promising delivery system for AONs.
\end{abstract}

KEYWORDS: oligonucleotide delivery, cell penetrating peptides, cellular internalization, cell surface proteoglycan binding peptide

\section{INTRODUCTION}

RNA interference mechanisms have attracted much attention in the recent decade, and advances in the design and development of oligonucleotides and drug delivery systems have rendered it possible to employ interfering RNAs for therapeutic purposes. Oligonucleotide therapeutics, which include antisense oligonucleotides (AONs), siRNAs (small interfering RNAs), CpG oligonucleotides, and aptamers, are a class of drugs noted for their exceptionally high target specificity facilitated by the base pairing between the target mRNA and the therapeutic oligonucleotide. Hence, oligonucleotide drugs overpower traditional small molecule drugs on account of the specifity of the binding, which is rendered by base pairing between target mRNA and therapeutic oligonucleotide. Within this class, AONs and siRNAs (expression modulating oligonucleotides) are short, synthetic nucleic acids designed to bind a target transcript and decrease the expression of the corresponding gene. The target gene is typically one that is overexpressed in a specific disease (cancer, cardiovascular, or metabolic), and its knock-down allows the diseased tissue to resume its normal function. ${ }^{2,3}$

Despite its relatively recent discovery, the simplicity and efficiency of the RNA interference process have led to the rapid proliferation of oligonucleotide-based drug candidates, and several clinical trials have been conducted to evaluate their feasibility for use as therapeutics. However, only two antisense oligonucleotide drugs have made it through the pipeline: Fomivirsen (Vitravene) and Mipomersen (Kynamro) were approved in 1998 and 2013, respectively. The remaining clinical trials were terminated without meeting their end points, principally as a result of inefficient biodistribution or poor pharmacodynamic and pharmacokinetic properties. ${ }^{4,5}$ In addition to the drawbacks regarding their in vivo efficiency, a second major obstacle in oligonucleotide delivery is the poor cell penetration capability of oligonucleotide-based drugs, which stems from their heavily negative charges. ${ }^{6}$ The negative charges of the oligonucleotide and the cell membrane act to repel the drug from the membrane, which complicates the mechanism of cellular internalization. Given the disadvantages of naked oligonucleotide delivery and the undesired properties of the free drug, an efficient delivery strategy is required for

Received: January 2, 2015

Revised: March 9, 2015

Accepted: March 31, 2015

Published: March 31, 2015 
oligonucleotide-mediated therapeutic efforts. Several delivery systems have been developed for this purpose using both viral delivery methods and nonviral delivery systems such as cationic lipids or cationic polymer systems. ${ }^{7}$ In the case of polymerbased carrier systems, the transfection efficiency of the cationic polymer was found to be correlated with its molecular weight, which is also correlated with carrier toxicity. In addition, polymers are synthesized with substantial variations in molecular weight, which complicates quality control and creates batch-to-batch variations. Cationic lipids also suffer from high toxicity due to their strongly positive charge. In response to the problems associated with cationic delivery systems, nontoxic liposomes (anionic and neutral liposomes) were proposed as alternative delivery agents, but the transfection efficiency of these liposomes and other noncationic systems (polymers, nanoparticles) were found to be too low for effective delivery. Consequently, the cellular internalization of these systems is often enhanced through surface modification methods. ${ }^{8}$

Cell-penetrating peptides (CPPs) are positively charged peptide sequences that facilitate the passage of their cargo through the cell membrane, and they are frequently used for the functionalization of drug carriers. There are two strategies for CPP-mediated oligonucleotide delivery based on covalent and noncovalent binding of the peptide to the oligonucleotide. Early studies were mostly based on the conjugation of the CPP to oligonucleotides in the form of peptide nucleic acids (PNA) and phosphorodiamidate morpholino oligomers (PMO). Noncovalent strategies, in contrast, turned out to be more advantageous not only due to their simplicity, but also for the low concentrations of oligonucleotide and CPP required for the system to be effective. CPP-functionalized drug delivery systems serve to protect the oligonucleotide from enzymatic degradation and allow usage of low doses of the oligonucleotide drug to reach therapeutic efficiency, which in turn decreases toxicity and off-target effects. ${ }^{9}$ Noncovalent functionalization of $\mathrm{TAT}^{10}{ }^{10}$ penetratin, ${ }^{11}$ and oligoarginine $\mathrm{CPPs}^{12,13}$ was studied for oligonucleotide and siRNA delivery. These systems are advantageous due to their high transfection efficiency; however, concerns were raised regarding the potential toxicity and nonspecificity of these carrier systems, especially in light of the findings that the CPPs in question may show toxic effects even at very low concentrations. ${ }^{14}$ It is therefore essential to design CPP-functionalized delivery systems to enhance membrane permeability while eliminating potential side effects. To this end, we designed a delivery system using peptide amphiphiles, bioinspired molecules that have the ability to self-assemble into a predetermined structure. The structures of peptide amphiphiles are highly variable, and this versatility allows them to be engineered for different purposes, facilitated through the use of peptide sequences known to play roles in different metabolic functions, such as membrane penetration, receptor binding, or proteoglycan binding. In this study, we designed self-assembling peptide amphiphiles incorporating two distinct types of bioactive peptide sequences: arginine rich cell penetrating peptides $\left(\mathrm{R}_{4}\right.$ and $\left.\mathrm{R}_{8}\right)$ and a cell surface proteoglycan binding peptide (KRSR). ${ }^{15}$ We then used these peptides decorated with two types of internalization-mediating signals to increase membrane penetration and the transfection efficiency of an oligonucleotide drug.

\section{MATERIALS AND METHODS}

Materials. 9-Fluorenylmethoxycarbonyl (Fmoc) and tertbutoxycarbonyl (Boc) protected amino acids, $\left[4-\left[\alpha-\left(2^{\prime}, 4^{\prime}\right.\right.\right.$ dimethoxyphenyl) Fmoc- aminomethyl] phenoxy]acetamidonorleucyl-MBHA resin (Rink amide MBHA resin), and 2-(1H-benzotriazol-1-yl)-1,1,3,3 tetramethyluronium hexafluorophosphate (HBTU) were purchased from NovaBiochem. Lauric acid and N,N-diisopropylethylamine (DIEA) were purchased from Merck. All other chemicals were purchased from Alfa Aesar and Sigma-Aldrich and used as provided. All water used was deionized water with a resistance of $18 \mathrm{M} \Omega \mathrm{cm}$ (Millipore Milli-Q). Dulbecco's Modified Eagle's Medium (DMEM), fetal bovine serum (FBS), penicillin-streptomycin (PS), and trypsin were purchased from Gibco, Life Technologies. Lipofectamine and Alamar Blue were purchased from Invitrogen; uptake inhibitors were purchased from Sigma. Bcl-2 antisense oligonucleotide (G-3129, Genasense) and fluorescein-6-amide (FAM) labeled antisense oligonucleotide having the sequence: 5'-TCTCCCAGCGTGCGCCAT-3' were donated by Genta Inc. G4126, the mismatch (MM) control with a sequence 5'-TCTCCCAGCATGTGCCAT-3', was used in expression studies.

Synthesis of Peptide Amphiphiles. Peptide amphiphiles were synthesized with the standard Fmoc solid phase peptide synthesis method. As a solid support, Rink Amide MBHA (0.59 mmol g ${ }^{-1}$ loading) resin was used. Dissolved Fmoc protected amino acid activated with HBTU, and DIEA was coupled to resin or previous amino acid for $3 \mathrm{~h}$. To monitor the completeness of coupling, Kaiser test was performed after each reaction. Deprotection of Fmoc protecting groups was done by adding $20 \%$ piperidine in DMF and allowing to shake for $20 \mathrm{~min}$. The resin was then treated with $10 \%$ acetic anhydride for $30 \mathrm{~min}$ to block unreacted amine groups. Peptide amphiphile was separated from the solid phase by using cleavage protocol where peptide was treated with trifluoroacetic acid (TFA)/triisopropylsilane (TIS)/water at a ratio of 95:2.5:2.5 for $2 \mathrm{~h}$. Peptide amphiphiles were precipitated with diethyl ether, and the precipitate was collected with centrifugation, dissolved in water, and freeze-dried. The peptides were characterized by reverse-phase high-performance liquid chromatography (HPLC) on an Agilent 6530 accurateMass Q-TOF LC/MS equipped with an Agilent 1200 HPLC. A Phenomenex Luna $3 \mu$ C8 100A $(50 \times 3.00 \mathrm{~mm})$ column and a water $(0.1 \%$ formic acid $) /$ acetonitrile $(0.1 \%$ formic acid $)$ gradient were used as stationary phase and mobile phase, respectively.

Circular Dichroism (CD). CD analysis was performed by J815 Jasco spectrophotometer with an integration time of $4 \mathrm{~s}$, bandwidth of $1 \mathrm{~nm}$, and data pitch of $0.1 \mathrm{~nm}$. PAs were dissolved in water to a final concentration of $200 \mu \mathrm{M}$. PA/AON samples were prepared to have a molar ratio of 30:1 PA/AON. Quartz cuvettes with $1 \mathrm{~mm}$ path lengths were used for measurements. An average of three readings were recorded; ellipticity was converted to molar ellipticity with the unit degree $\mathrm{cm}^{2} \mathrm{dmol}^{-1}$.

Transmission Electron Microscopy (TEM). PA samples were prepared with $0.05 \%(\mathrm{w} / \mathrm{v}) \mathrm{PA}$ solution, and PA/AON complexes were prepared to have final concentrations of $0.05 \%$ $(\mathrm{w} / \mathrm{v}) \mathrm{PA}$ to $50 \mathrm{ng} / \mu \mathrm{L}$ AON. Solutions were dropped onto 200-mesh copper grids. After $5 \mathrm{~min}$, samples were removed, and grids were stained with $2 \%$ uranyl acetate for $3 \mathrm{~min}$. After 
a)

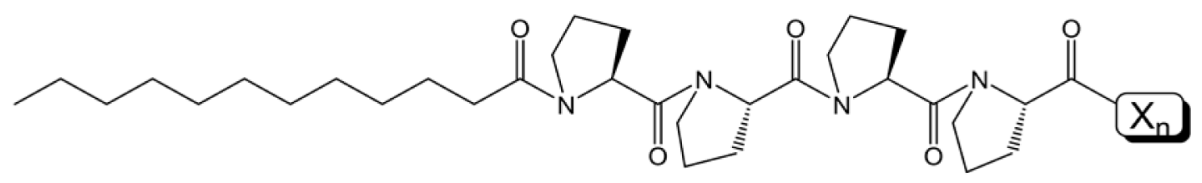

K-PA $\quad X_{1}=$ Gly-Lys-Am

$\mathbf{R}_{\mathbf{4}}$-PA $\quad \mathrm{X}_{3}=(\operatorname{Arg})_{4}-\mathrm{Am}$

KRSR-PA $\quad X_{2}=$ Gly-Lys-Arg-Ser-Arg-Am

$\mathbf{R}_{\mathbf{8}}$-PA $\quad \mathrm{X}_{4}=(\mathrm{Lys})_{2}(\mathrm{Arg})_{8}-\mathrm{Am}$

b)

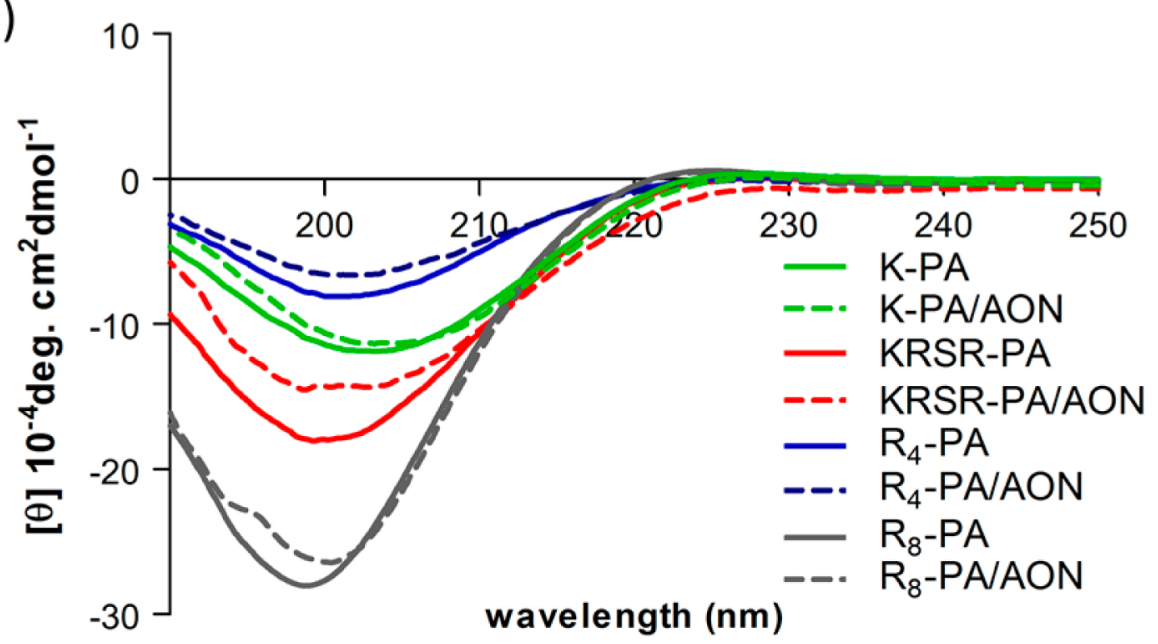

Figure 1. (a) Chemical representation of PAs used in this study: lauryl- $\mathrm{P}_{4} \mathrm{GK}-\mathrm{Am}$ (K-PA), lauryl- $\mathrm{P}_{4}$ GKRSR-Am (KRSR-PA), lauryl- $\mathrm{P}_{4} \mathrm{R}_{4}$-Am ( $\mathrm{R}_{4}$ $\mathrm{PA})$, and lauryl- $\mathrm{P}_{4} \mathrm{~K}_{2} \mathrm{R}_{8}-\mathrm{Am}\left(\mathrm{R}_{8}-\mathrm{PA}\right)$. (b) Circular dichroism spectra of PAs and PA/AON complexes.

washing, samples were air-dried, and images were obtained by a FEI Tecnai G2 F30 TEM at $200 \mathrm{keV}$.

Maintenance of Cells and Transfection with PA/AON Complexes. MCF-7 cells were maintained in DMEM with $10 \%$ FBS and 1\% PS and passaged at cell confluency between 80 and $90 \%$ using trypsin-ethylenediaminetetraacetic acid (EDTA). PA/AON complexes were prepared in water for AON transfection to have molar ratios of 30:1 (PA/AON). MCF-7 cell line was used as a breast cancer cell model for delivering Bcl-2 antisense oligonucleotide.

Cellular Internalization of PA/AON Complexes. For confocal microscopy, $4 \times 10^{4} \mathrm{MCF}-7$ cells per well were seeded in 24-well plates in standard medium. After $24 \mathrm{~h}$, medium was discarded, serum-free medium was added, and PA/AON complexes were administered to have a final concentration of $30 \mu \mathrm{M}$ PA and $1 \mu \mathrm{M}$ AON. After $24 \mathrm{~h}$ of incubation, cells were washed with phosphate-buffered saline (PBS) three times, fixed with $4 \%$ paraformaldehyde (PFA), and stained with phalloidin and TO-PRO-3. Cells were then visualized by a laser scanning confocal microscope (LSM 510, Zeiss).

Flow Cytometry. For flow cytometry, $2 \times 10^{4} \mathrm{MCF}-7$ cells per well were seeded in 96-well plates with standard medium. After $24 \mathrm{~h}$ of cell seeding, medium was changed to serum-free medium. PA/AON complexes were prepared and administered to have final concentrations of $30 \mu \mathrm{M}$ PA and $1 \mu \mathrm{M}$ AON. Cells were incubated for $24 \mathrm{~h}$ and suspended by trypsinization. After washing twice with PBS and centrifugation at $1500 \mathrm{rpm}$ for $5 \mathrm{~min}$, cells were resuspended in PBS. Cells were analyzed by a Guava easyCyte (Milipore) flow cytometer. By using nontreated control, cells were gated by SSC (side scatter channel) and FSC (forward scatter channel). Fluorescence intensity of FAM-AON was measured by the green channel.

Mechanisms of Internalization. To study the internalization mechanism of $\mathrm{PA} / \mathrm{AON}$ complexes, chemical inhibitors were used to inhibit specific internalization pathways. Aliquots of $2 \times 10^{4}$ MCF-7 cells per well were seeded in 96-well plates with standard medium. After $24 \mathrm{~h}$ of seeding, medium was changed to serum-free medium, and inhibitor solutions were administered at final concentrations of $100 \mu \mathrm{M}$ amiloride, 20 $\mu \mathrm{M}$ dynasore, $0.2 \mu \mathrm{g} / \mathrm{mL}$ chlorpromazine, and $10 \mu \mathrm{g} / \mathrm{mL}$ nystatin. After $1 \mathrm{~h}$ of incubation with inhibitors, PA/AON complexes were prepared and administered at final concentrations of $30 \mu \mathrm{M}$ PA and $1 \mu \mathrm{M}$ AON. Cells were incubated for $24 \mathrm{~h}$ and suspended with trypsinization. After being washed with PBS twice, cells were collected and analyzed with a flow cytometer as described above.

Gene Expression Analyses. Aliquots of $4 \times 10^{5}$ MCF-7 cells per well were seeded in 6-well plates with standard medium. After $24 \mathrm{~h}$, medium was discarded, and PA/AON and $\mathrm{PA} / \mathrm{MM}$ complexes were administered in serum-free medium at final concentrations of $60 \mu \mathrm{M}$ PA and $2 \mu \mathrm{M}$ oligonucleotide. Complexes were incubated for $24 \mathrm{~h}$, except $\mathrm{R}_{8}-\mathrm{PA}$, which was administered at $20 \mu \mathrm{M}$ concentration and incubated for $4 \mathrm{~h}$ due to its toxicity. Lipofectamine control, AON-only, and MM groups were also administered at a final concentration of $2 \mu \mathrm{M}$ oligonucleotide. Lipofectamine was used according to the manufacturer's instructions. After incubation, cells were washed with PBS, and RNA was extracted with TRIzol reagent. Expression levels of Bcl-2 were investigated by a Biorad C1000 thermal cycler using a one step qRT-PCR kit (Invitrogen). Expression levels were normalized to GAPDH. 

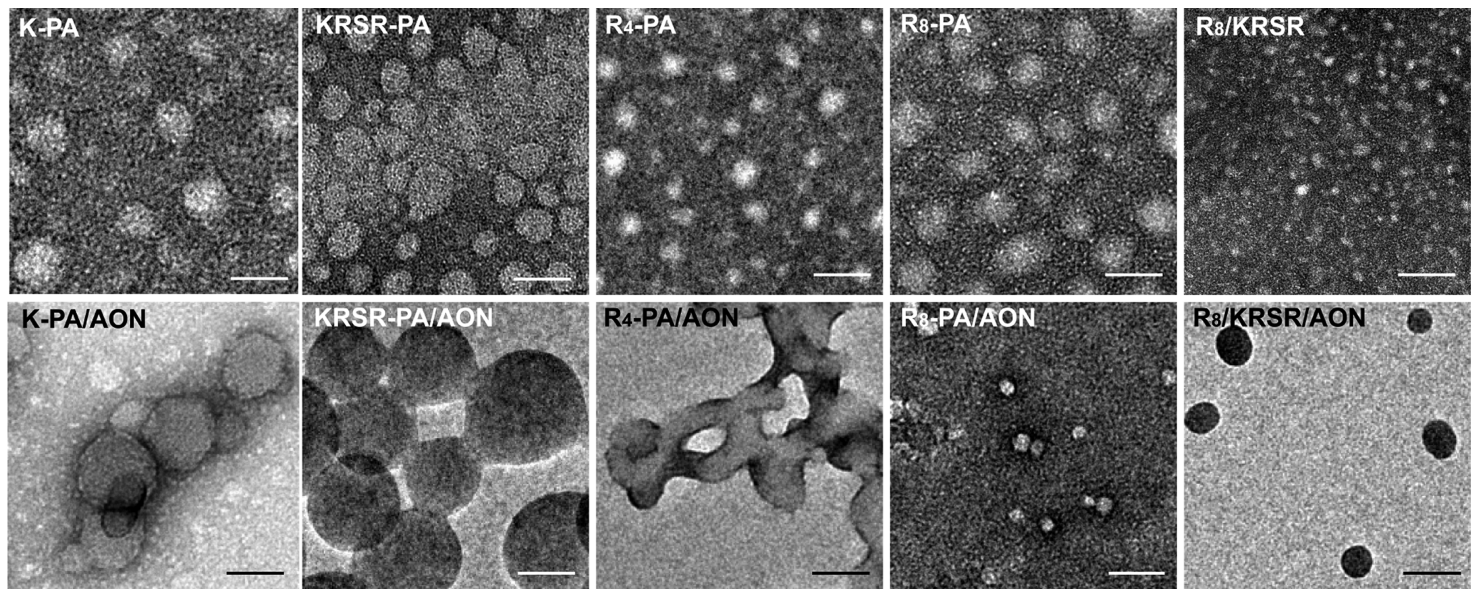

Figure 2. TEM images of PAs and PA/AON complexes (scale bars: $50 \mathrm{~nm}$ ).

\section{RESULTS AND DISCUSSION}

Design of Peptide Amphiphiles. Peptide amphiphiles were designed with an alkyl tail, a proline-rich sequence, and a bioactive unit (Figure 1). Proline residues, known as $\beta$-sheet breakers, were used to force the final self-assembled structure to have a spherical shape. ${ }^{16}$ The driving forces for self-assembly were $\mathrm{H}$-bonding and hydrophobic and electrostatic interactions. As a result of the self-assembly process, the hydrophobic alkyl tails were buried to the interior of the structure, and the bioactive peptide sequences were presented on the exterior. Peptide amphiphiles were decorated with two different polyarginine peptides, tetraarginine $\left(\mathrm{R}_{4}\right)$ and octaarginine $\left(\mathrm{R}_{8}\right)$, to enhance their cellular internalization. Arginine-rich cell-penetrating peptides were shown to be effective in cargo delivery in numerous studies. ${ }^{17}$ On the basis of the knowledge that the peptide-proteoglycan interactions mediate cellular internalization, ${ }^{18}$ in addition to polyarginine residues, we designed our cell surface-binding peptide with the sequence KRSR, which follows the general heparin-binding sequence BBXB (the Cardin-Weintraub motif). This sequence was found to be consensus in heparin binding peptides and proteins ${ }^{15}$ and has been proposed as a potential common-receptor binding peptide for drug delivery. ${ }^{19}$ In addition to the proteoglycan binding propensity of KRSR peptide, its basic nature also fortifies the binding to the cell surface membrane and enables translocation of the cargo similarly to other cell-penetrating peptides. K-PA was used as a nonbioactive PA control. Solid phase peptide synthesis method was used to synthesize all peptide amphiphiles, and they were analyzed by liquid chromatography and mass spectrometry (Figure S1, Supporting Information).

Characterization of the PA/AON Complexes. The secondary structure, morphology, size, and zeta potential of peptide amphiphiles and peptide amphiphile-oligonucleotide complexes were characterized to gain insight into their biophysical, morphological, and physical features. Secondary structures of PAs were determined by CD (Figure 1). All PAs and PA/AON complexes displayed a negative peak around 200 $\mathrm{nm}$, which revealed that they exhibited random coil structure. ${ }^{16}$

The morphology of PAs and PA/AON complexes was observed by TEM imaging (Figure 2). According to TEM images, all bare peptide amphiphiles formed spherical nanostructures. Their sizes varied between 15 and $45 \mathrm{~nm}$ depending on the peptide sequence except $\mathrm{R}_{8} / \mathrm{KRSR}$ mixture.
Upon complexation with AON, there was a tendency for the nanospherical particles to increase their size, especially in the $\mathrm{R}_{4}-\mathrm{PA} / \mathrm{AON}$ and KRSR-PA/AON samples. The size increase might be explained by the fusion of peptide amphiphile micelles with oligonucleotides, or these structures might be micellar aggregates of peptide amphiphiles formed due to the presence of oligonucleotide. $\mathrm{R}_{4}$-PA formed more elongated spheres in the presence of $\mathrm{AON}$, which were typically clustered together. Compared to $\mathrm{R}_{4}-\mathrm{PA}$, KRSR-PA/AON formed more regular spherical structures that did not aggregate. In contrast to the general tendency to increase in size, $\mathrm{R}_{8}$-PA formed smaller nanospherical structures when complexed with AONs. Since each PA contains a difference sequence, the size changes observed in AON-complexed PAs are attributed to the variance in PA-AON interactions, which are expected to be mediated primarily through the peptide moieties.

The hydrodynamic sizes of peptide amphiphiles were investigated with dynamic light scattering measurements, and the unmodified PA size was found to be around $70 \mathrm{~nm}$ in diameter, which is greater than the sizes in TEM images (Figure S2, Supporting Information). The discrepancy in size measurements can be attributed to the differences between the two measurement methods. While dried samples were used for TEM imaging, which may cause shrinking of the micelles, dynamic light scattering measures the size of highly hydrated micelles. ${ }^{20}$ When PAs were complexed with AON, K-PA/AON, $\mathrm{R}_{8}-\mathrm{PA} / \mathrm{AON}$, and $\mathrm{R}_{8} / \mathrm{KRSR} / \mathrm{AON}$, they formed similarly sized, small spherical nanostructures around $70 \mathrm{~nm}$ in diameter, whereas complexation with AON increased the size of KRSR$\mathrm{PA}$ and $\mathrm{R}_{4}-\mathrm{PA} / \mathrm{AON}$, which was also the case in transmission electron micrographs.

Zeta potential of the bare PA and PA/AON complexes was measured by using Zetasizer, and the results are provided in Table S1 of the Supporting Information. Unmodified peptide amphiphiles were positively charged as a result of the presence of lysine and arginine residues. The design of PAs was based on the idea that introducing positive charges to the system would facilitate complexation between the PAs and the AONs through electrostatic interactions. The charge of K-PA, which contains only one lysine as a basic amino acid, was slightly positive. PAs containing more than one basic amino acid ( $\mathrm{R}_{4}$-PA, KRSR-PA, and $\mathrm{R}_{8}-\mathrm{PA}$ ) had greater zeta potentials, while $\mathrm{R}_{8}-\mathrm{PA}$, with its octoarginine residue, had the highest zeta potential. After complexation with AON, zeta potentials of PAs decreased, 

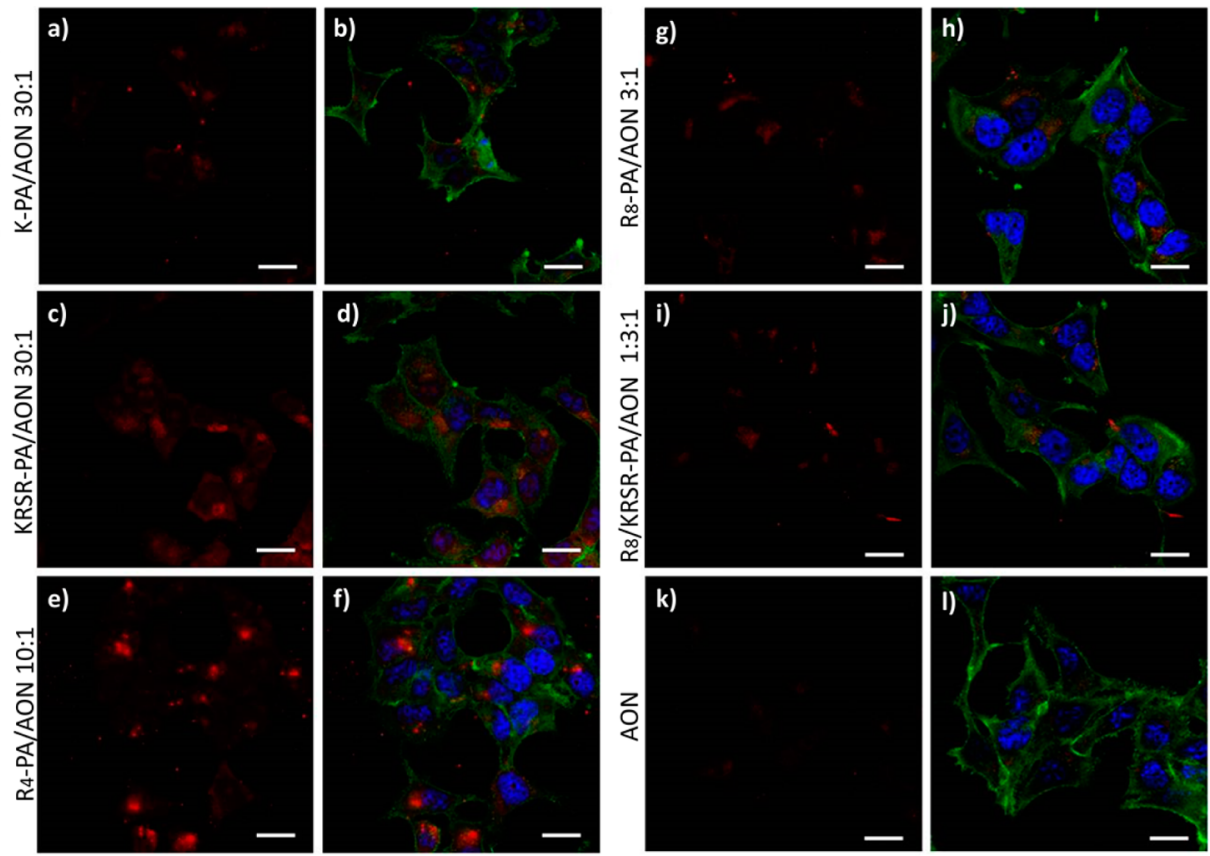

Figure 3. Confocal fluorescence images of MCF-7 cells treated with PA/AON complexes after $24 \mathrm{~h}$. MCF-7 cells treated with PA/AON complexes (a) K-PA/AON, (c) KRSR-PA/AON, (e) $\mathrm{R}_{4}-\mathrm{PA} / \mathrm{AON}$, (g) $\mathrm{R}_{8}-\mathrm{PA} / \mathrm{AON}$, (i) $\mathrm{R}_{8} / \mathrm{KRSR}-\mathrm{PA} / \mathrm{AON}$, (k) AON. Merged images of PA/AON complexes and cells stained with phalloidin and TO-PRO-3 (b) K-PA/AON, (d) KRSR-PA/AON, (f) R-PA/AON, (h) R8-PA/AON, (j) R 8 /KRSRPA/AON, (l) AON. FAM-AON (red), actins (green), nuclei (blue) (scale bars: $20 \mu \mathrm{m}$ ).

likely due to the negative charge imparted by the oligonucleotide itself.

Biocompatibility of the PA and PA/AON Systems. The viability of MCF-7 cells incubated with PAs was investigated after $24 \mathrm{~h}$ (Figure S3, Supporting Information). K-PA, KRSR$\mathrm{PA}$, and $\mathrm{R}_{4}$-PA were found to be biocompatible at $200 \mu \mathrm{M}$, which was far above the effective dose $(30-60 \mu \mathrm{M})$ used for our in vitro experiments. $\mathrm{R}_{8}-\mathrm{PA}$, on the other hand, was cytotoxic at $50 \mu \mathrm{M}$. Polyarginine CPPs are known to be internalized more as the number of arginines in the sequence is increased, which in turn increases the toxicity of the peptide. ${ }^{21}$ Toxicity therefore depends on the arginine number, cell type, and incubation period. In general, the $\mathrm{IC}_{50}$ value of polyarginines with more than eight arginines in their sequence was found to be between 10 and $100 \mu \mathrm{M}^{21,22}$ for short incubation times. In the present study, we found the $\mathrm{IC}_{50}$ of peptide amphiphile containing $\mathrm{R}_{8}$ to be around $50 \mu \mathrm{M}$ after 24 $h$ of incubation, which indicates that the toxicity of $\mathrm{R}_{8}$-PA stems from the presence of oligoarginine in the sequence.

Cellular Internalization Analyses. The cellular internalization of $\mathrm{PA} / \mathrm{AON}$ complexes was monitored by confocal microscopy after $24 \mathrm{~h}$ of incubation (Figure 3 ). The internalizations of KRSR-PA/AON (Figure 3c,d), $\mathrm{R}_{4}$-PA/ $\mathrm{AON}$ (Figure 3e,f), and $\mathrm{R}_{8}-\mathrm{PA} / \mathrm{AON}$ (Figure $3 \mathrm{~g}, \mathrm{~h}$ ) were observed to be more effective compared to K-PA/AON (Figure $3 a, b)$. The internalization of $R_{8}-P A / A O N$ was significantly greater than control even at low concentrations of $\mathrm{R}_{8}$-PA (3 $\mu \mathrm{M}$ ). The internalization of $\mathrm{R}_{8} / \mathrm{KRSR} / \mathrm{AON}$ complex (Figure $3 \mathrm{i}, \mathrm{j})$ containing $1 \mu \mathrm{M} \mathrm{R} \mathrm{R}_{8} \mathrm{PA}$ and $3 \mu \mathrm{M}$ KRSR-PA was also significantly greater than the control. The combination of the two PA molecules, one binding to cell surface heparins and the other penetrating the cell membrane, was therefore observed to be more effective for cellular internalization.

The uptake was also quantified by flow cytometry, in which the fluorescence of FAM-AON was quantified in MCF-7 cells incubated with $\mathrm{PA} / \mathrm{AON}$ complexes for $24 \mathrm{~h}$ (Figure 4). The internalization of KRSR-PA/AON and $\mathrm{R}_{4}-\mathrm{PA} / \mathrm{AON}$ was found

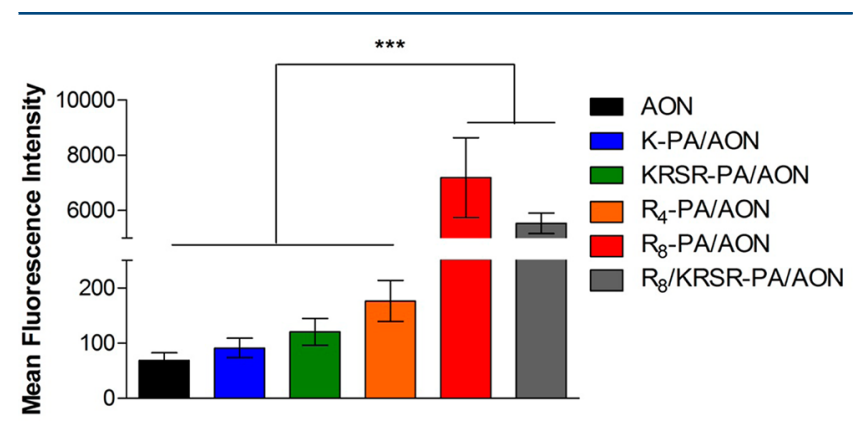

Figure 4. Uptake of PA/AON complexes by MCF-7 cells quantified with flow cytometry. (Error bars show SEM; two independent experiments were repeated with $n=3$ in each experiment. One-way ANOVA shows statistical significance with $* p<0.05, * * p<0.01$, $* * * p<0.001$.)

to be slightly increased compared to K-PA. The uptake of $\mathrm{R}_{8}$ $\mathrm{PA} / \mathrm{AON}$ complex, administered at the same concentration with other PA/AON complexes, was particularly drastic. When $\mathrm{R}_{8}$-PA and $\mathrm{R}_{4}$-PA were compared, cellular internalization was observed to increase as the number of arginines in the sequence increased, which is consistent with previous results. ${ }^{21}$

Interestingly, the internalization efficiency of the combination of $\mathrm{R}_{8} / \mathrm{KRSR} / \mathrm{AON}$ was almost the same as $\mathrm{R}_{8}-\mathrm{PA} / \mathrm{AON}$, although a lower amount of $\mathrm{R}_{8}-\mathrm{PA}$ was present. Thus, by combining the noncytotoxic properties of KRSR-PA with the high internalization capacity of $\mathrm{R}_{8}-\mathrm{PA}$, the combination of $\mathrm{R}_{8}$ $\mathrm{PA}$ and KRSR-PA might be an effective oligonucleotide delivery system with its remarkable internalization efficiency.

Mechanisms of Cellular Internalization. The internalization mechanism of polyarginine-functionalized delivery 

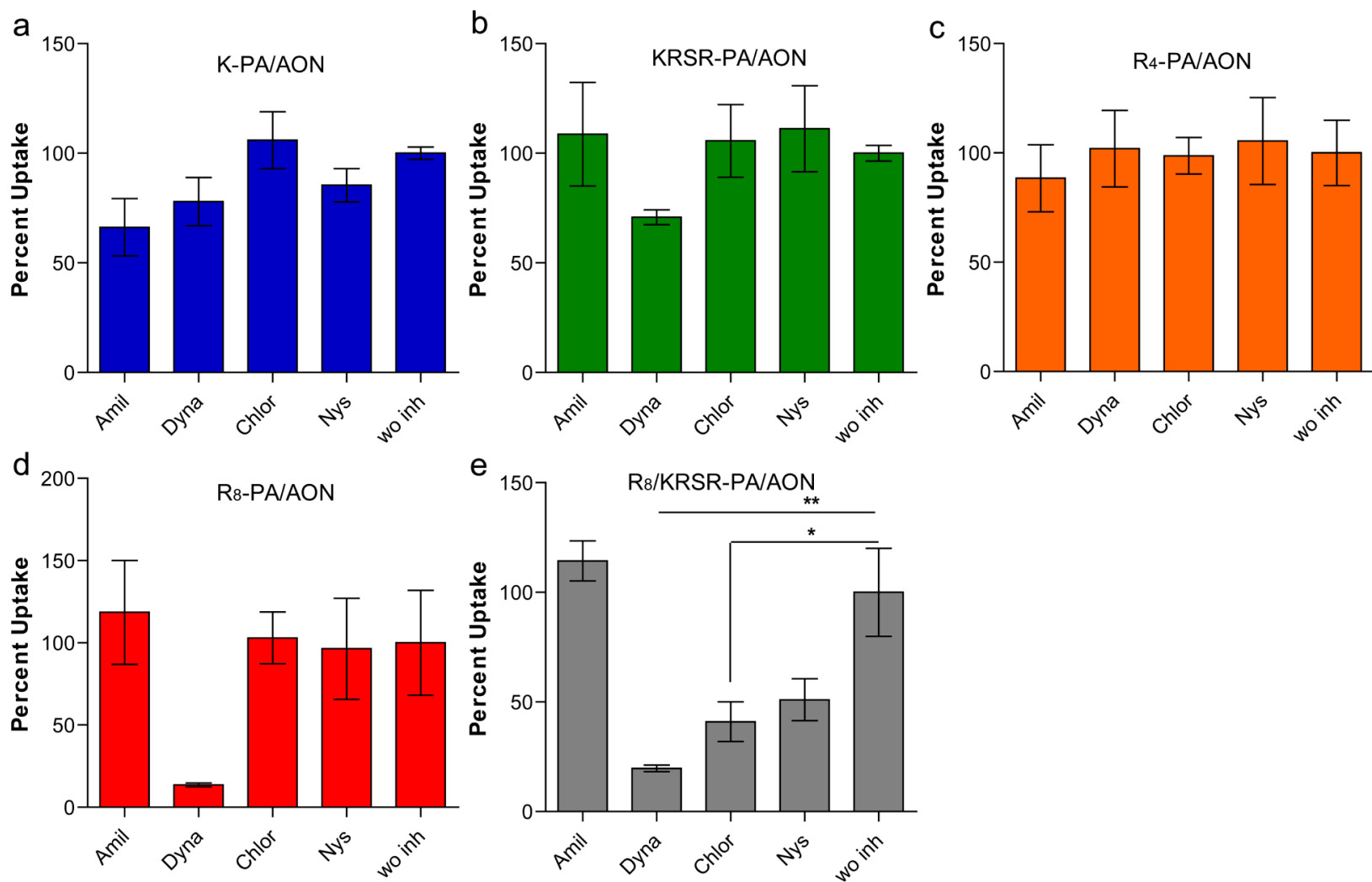

Figure 5. Mechanisms of cellular internalization of PA/AON complexes in MCF-7 cells. Amiloride (Amil), Dynasore (Dyna), Chlorpromazine (Chlor), and Nystatin (Nys) were administered to cells to inhibit endocytosis pathways; uptake was normalized to cells without inhibitor (w/o inh). (Error bars show SEM; two independent experiments were repeated with $n=3$ in each experiment. One-way ANOVA shows statistical significance with $* p<0.05, * * p<0.01, * * * p<0.001$.)

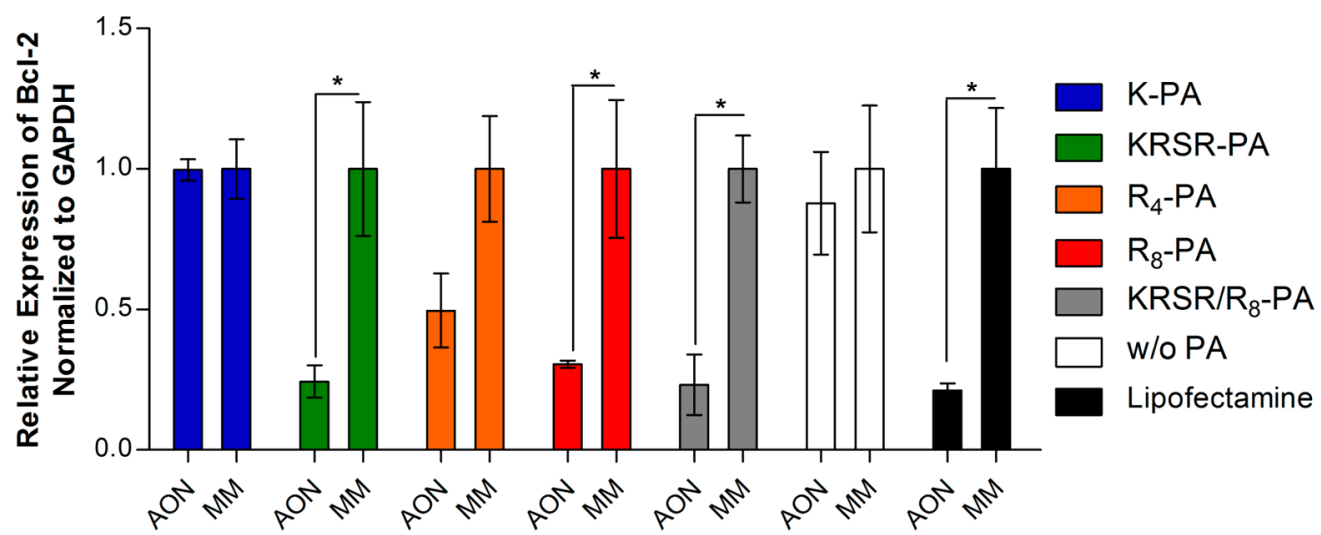

Figure 6. Bcl-2 expression in MCF-7 cells treated with PA/AON or PA/MM complexes. Gene expression was normalized to GAPDH. AON, antisense oligonucleotide; MM, mismatch control. (Error bars show SEM; two independent experiments were repeated with $n=3$ in each experiment. Student's $t$ test shows statistical significance with $* p<0.05, * * p<0.01, * * * p<0.001$.)

systems has been controversial; however, it has recently been reported that the pathway utilized mostly depends on the concentration of the peptide. ${ }^{23}$ Previously, direct translocation of arginine rich peptides was thought to be the main mechanism, ${ }^{24}$ yet peptides were demonstrated to be taken up by endocytosis at low concentrations (below $10 \mu \mathrm{M}$ ). ${ }^{25}$ At high concentrations, however, the endocytosis-independent mode of uptake (rapid nuclear zone dependent uptake) was observed to be the dominant mechanism. This mode of uptake was reported to be dependent on the dynamin and heparan sulfate residues on the cell surface. ${ }^{25}$
Uptake inhibitors were used to study the mechanisms involved in the internalization of PAs decorated with CPPs and heparin binding sequences (Figure 5). Amiloride, dynasore, chlorpromazine, and nystatin were used to inhibit macropinocytosis, dynamin-dependent pathway, clathrin-mediated endocytosis, and caveolae-mediated endocytosis, respectively. ${ }^{26}$ The internalization of K-PA and $\mathrm{R}_{4}$-PA was not inhibited with any of the chemical inhibitors used, which strongly indicates that the internalization was via a nonendocytotic pathway and probably through direct translocation. KRSR-PA and $\mathrm{R}_{8}-\mathrm{PA}$ internalization, unlike other PAs, was found to be dynaminmediated at $30 \mu \mathrm{M} \mathrm{PA}$ concentration. Interestingly, the $\mathrm{R}_{8} /$ 
KRSR-PA complex was demonstrated to be internalized via clathrin- and caveolae-mediated endocytosis and dynamindependent pathway. Overall, these results demonstrate that the amino acid sequences presented on the surface of PAs are critical in modulating the uptake mechanism of $\mathrm{PA} / \mathrm{AON}$ complexes.

Gene Expression Analyses. An efficient delivery vehicle must carry the oligonucleotide into the cell, escape from endosome, and carry the molecule to the target organelle. Antisense oligonucleotides (except splice-correction AONs) bind to target mRNAs in the cytosol to induce translational arrest. Therefore, $\mathrm{Bcl}-2 \mathrm{AON}$ would silence $\mathrm{Bcl}-2$ gene expression only in the presence of an efficient transfection system that delivers the intact AON into the cytosol. Bcl-2 expression following $\mathrm{PA} / \mathrm{AON}$ complex administration was investigated in MCF-7 cells, and all PA/AON complexes except $\mathrm{K}-\mathrm{PA} / \mathrm{AON}$ were shown to be effective in decreasing the gene expression (Figure 6). KRSR-PA/AON, $\mathrm{R}_{8}-\mathrm{PA} / \mathrm{AON}$, and the combination of these two PAs significantly silenced the Bcl-2 gene in MCF-7 cells. The expression decrease in KRSR-PA/ $\mathrm{AON}, \mathrm{R}_{8}-\mathrm{PA} / \mathrm{AON}$, and the combination was comparable to the lipofectamine control, which is a commercial transfection agent with a cationic lipid composition. Although the cellular internalizations of KRSR-PA/AON and $\mathrm{R}_{4}$-PA were only slightly higher compared to K-PA, delivery with K-PA did not induce Bcl-2 silencing, which suggests that K-PA could not provide endosomal escape, whereas KRSR-PA and $\mathrm{R}_{4}-\mathrm{PA}$ could. Endosomal escape, which can be facilitated by the multivalency of the CPPs, ${ }^{27}$ is a prerequisite for $\mathrm{AONs}$ to be functional. Our PA delivery system presenting multivalent CPPs on the surface is an example of this strategy, which allows $\mathrm{CPP}$-decorated PAs to induce the endosomal escape and thus protect the functionality of their AON cargo.

\section{CONCLUSION}

Here we designed a bioinspired, biodegradable, and bioactive oligonucleotide delivery system with self-assembling PAs. The system was decorated with cell penetrating peptides and a cell surface binding peptide to increase the cellular internalization of the oligonucleotide. All bioactive PAs were shown to have high transfection efficiencies, yet the cell surface binding KRSR$\mathrm{PA}$ is outstanding compared to other delivery systems in providing both high transfection efficiency and low toxicity. Considering its advantages over other delivery systems, the designed carrier system holds promise for oligonucleotide delivery, especially with regards to its ability to self-assemble into nanostructures and present multivalent CPPs on the surface through self-assembly. These bioinspired self-assembling systems can provide transfection efficiencies comparable to viral delivery without contamination risk of viral proteins. These designs can also supersede polymer, lipid, and nanoparticle-based delivery systems owing to their practical properties such as simple design and synthesis, facile functionalization, and high biocompatibility.

\section{ASSOCIATED CONTENT}

\section{S Supporting Information}

Liquid chromatogram and mass spectra of PAs, size distribution of $\mathrm{PA}$ and $\mathrm{AON} / \mathrm{PA}$ nanospheres, cytotoxicity of PAs administered to MCF7 cells for $24 \mathrm{~h}$, zeta potentials of PA and AON/PA complexes, zeta potentials of $\mathrm{R}_{8} / \mathrm{KRSR}-\mathrm{PA} /$ AON complexes. This material is available free of charge via the Internet at http://pubs.acs.org.

\section{AUTHOR INFORMATION}

\section{Corresponding Authors}

*E-mail: moguler@unam.bilkent.edu.tr.

*E-mail: atekinay@unam.bilkent.edu.tr. Phone: +90 312290 3572. Fax: +90 3122664365.

\section{Notes}

The authors declare no competing financial interest.

\section{ACKNOWLEDGMENTS}

D.M. and M.S. are supported by TUBITAK-BIDEB fellowship. M.O.G. and A.B.T. acknowledge support from the Turkish Academy of Sciences Distinguished Young Scientist Award (TUBA-GEBIP).

\section{REFERENCES}

(1) Bennett, C. F.; Swayze, E. E. RNA Targeting Therapeutics: Molecular Mechanisms of Antisense Oligonucleotides as a Therapeutic Platform. Annu. Rev. Pharmacol. 2010, 50, 259-293.

(2) Kole, R.; Krainer, A. R.; Altman, S. RNA Therapeutics: Beyond RNA Interference and Antisense Oligonucleotides. Nat. Rev. Drug Discovery 2012, 11 (2), 125-140.

(3) Agrawal, N.; Dasaradhi, P. V. N.; Mohmmed, A.; Malhotra, P.; Bhatnagar, R. K.; Mukherjee, S. K. RNA Interference: Biology, Mechanism, and Applications. Microbiol. Mol. Biol. Rev. 2003, 67 (4), 657.

(4) Juliano, R.; Bauman, J.; Kang, H.; Ming, X. Biological Barriers to Therapy with Antisense and siRNA Oligonucleotides. Mol. Pharmaceutics 2009, 6 (3), 686-695.

(5) Yu, B.; Zhao, X.; Lee, L. J.; Lee, R. J. Targeted Delivery Systems for Oligonucleotide Therapeutics. AAPS J. 2009, 11 (1), 195-203.

(6) Dias, N.; Stein, C. A. Antisense Oligonucleotides: Basic Concepts and Mechanisms. Mol. Cancer Ther. 2002, 1 (5), 347-355.

(7) Zhu, L.; Mahato, R. I. Lipid and Polymeric Carrier-Mediated Nucleic Acid Delivery. Expert Opin. Drug Delivery 2010, 7 (10), 12091226.

(8) Stewart, K. M.; Horton, K. L.; Kelley, S. O. Cell-Penetrating Peptides As Delivery Vehicles for Biology and Medicine. Org. Biomol. Chem. 2008, 6 (13), 2242-2255.

(9) Margus, H.; Padari, K.; Pooga, M. Cell-Penetrating Peptides as Versatile Vehicles for Oligonucleotide Delivery. Mol. Ther. 2012, 20 (3), 525-533.

(10) Kang, H. M.; DeLong, R.; Fisher, M. H.; Juliano, R. L. TatConjugated PAMAM Dendrimers As Delivery Agents for Antisense and siRNA Oligonucleotides. Pharm. Res. 2005, 22 (12), 2099-2106.

(11) Lundberg, P.; El-Andaloussi, S.; Sutlu, T.; Johansson, H.; Langel, U. Delivery of Short Interfering RNA Using Endosomolytic Cell-Penetrating Peptides. FASEB J. 2007, 21 (11), 2664-2671.

(12) Kim, W. L.; Christensen, L. V.; Jo, S.; Yockman, J. W.; Jeong, J. H.; Kim, Y. H.; Kim, S. W. Cholesteryl Oligoarginine Delivering Vascular Endothelial Growth Factor siRNA Effectively Inhibits Tumor Growth in Colon Adenocarcinoma. Mol. Ther. 2006, 14 (3), 343-350.

(13) Kumar, P.; Wu, H. Q.; McBride, J. L.; Jung, K. E.; Kim, M. H.; Davidson, B. L.; Lee, S. K.; Shankar, P.; Manjunath, N. Transvascular Delivery of Small Interfering RNA to the Central Nervous System. Nature 2007, 448 (7149), 39-43.

(14) Lee, S. H.; Castagner, B.; Leroux, J.-C. Is There a Future for Cell-Penetrating Peptides in Oligonucleotide Delivery? Eur. J. Pharm. Biopharm. 2013, 85 (1), 5-11.

(15) Cardin, A. D.; Weintraub, H. J. R. Molecular Modeling of Protein-Glycosaminoglycan Interactions. Arteriosclerosis 1989, 9 (1), $21-32$.

(16) Guler, M. O.; Claussen, R. C.; Stupp, S. I. Encapsulation of Pyrene within Self-Assembled Peptide Amphiphile Nanofibers. J. Mater. Chem. 2005, 15 (42), 4507-4512.

(17) Margus, H.; Padari, K.; Pooga, M. Cell-Penetrating Peptides as Versatile Vehicles for Oligonucleotide Delivery. Mol. Ther. 2012, 20 (3), 525-533. 
(18) Wallbrecher, R.; Verdurmen, W. P.; Schmidt, S.; Bovee-Geurts, P. H.; Broecker, F.; Reinhardt, A.; van Kuppevelt, T. H.; Seeberger, P. H.; Brock, R. The Stoichiometry of Peptide-Heparan Sulfate Binding as a Determinant of Uptake Efficiency of Cell-Penetrating Peptides. Cell. Mol. Life Sci. 2013, 1-13.

(19) Stephenson, V. C.; Heyding, R. A.; Weaver, D. F. The "Promiscuous Drug Concept" With Applications to Alzheimer's Disease. FEBS Lett. 2005, 579 (6), 1338-1342.

(20) Wang, Y.; Wang, Y. N.; Wu, G. L.; Fan, Y. G.; Ma, J. B. pHResponsive Self-Assembly and Conformational Transition of Partially Propyl-Esterified Poly(alpha,beta-L-aspartic acid) as Amphiphilic Biodegradable Polyanion. Colloids Surf., B 2009, 68 (1), 13-19.

(21) Tunnemann, G.; Ter-Avetisyan, G.; Martin, R. M.; Stockl, M.; Herrmann, A.; Cardoso, M. C. Live-Cell Analysis of Cell Penetration Ability and Toxicity of Oligo-Arginines. J. Pept. Sci. 2008, 14 (4), 46976.

(22) Jones, S. W.; Christison, R.; Bundell, K.; Voyce, C. J.; Brockbank, S. M.; Newham, P.; Lindsay, M. A. Characterisation of Cell-Penetrating Peptide-Mediated Peptide Delivery. Br. J. Pharmacol. 2005, 145 (8), 1093-102.

(23) Brock, R. The Uptake of Arginine-Rich Cell-Penetrating Peptides: Putting the Puzzle Together. Bioconjugate Chem. 2014, 25 (5), 863-868.

(24) Futaki, S.; Goto, S.; Sugiura, Y. Membrane Permeability Commonly Shared among Arginine-Rich Peptides. J. Mol. Recognit. 2003, 16 (5), 260-264.

(25) Duchardt, F.; Fotin-Mleczek, M.; Schwarz, H.; Fischer, R; Brock, R. A Comprehensive Model for the Cellular Uptake of Cationic Cell-Penetrating Peptides. Traffic 2007, 8 (7), 848-66.

(26) Malefyt, A. P.; Walton, S. P.; Chan, C. Endocytosis Pathways for Nucleic Acid Therapeutics. Nano Life 2012, 2 (3), 1241005.

(27) Erazo-Oliveras, A.; Muthukrishnan, N.; Baker, R.; Wang, T. Y.; Pellois, J. P. Improving the Endosomal Escape of Cell-Penetrating Peptides and Their Cargos: Strategies and Challenges. Pharmaceuticals (Basel) 2012, 5 (11), 1177-1209. 